\title{
A one-shot inpainting algorithm based on the topological asymptotic analysis
}

\author{
DIDIER AUROUX* and MOHAMED MASMOUDI \\ Laboratoire MIP, Université Paul Sabatier Toulouse 3, 31062 Toulouse cedex 9, France \\ *E-mail: auroux@mip.ups-tlse.fr
}

\begin{abstract}
The aim of this article is to propose a new method for the inpainting problem. Inpainting is the problem of filling-in holes in images. We consider in this article the crack localization problem, which can be solved using the Dirichlet to Neumann approach and the topological gradient. In a similar way, we can define a Dirichlet and a Neumann inpainting problem. We then define a cost function measuring the discrepancy between the two corresponding solutions. The minimization is done using the topological asymptotic analysis, and is performed in only one iteration. The optimal solution provides the best localization of the missing edges, and it is then easy to inpaint the holes.
\end{abstract}

Mathematical subject classification: $68 \mathrm{U} 10,94 \mathrm{~A} 08,34 \mathrm{E} 05$.

Key words: image inpainting, topological asymptotic analysis, inverse conductivity problem.

\section{Introduction}

The inverse conductivity problem, the so called Calderon problem [12], consists in identifying the coefficients of a partial differential equation from the knowledge of the Dirichlet to Neumann operator. This problem has been widely studied in literature [15, 16, 23, 24].

In the particular case of cracks identification, the problem seems to be more convenient to solve thanks to the singularities of the solution. Only two measurements are needed to recover several simple cracks $[2,7,3]$. 
From the numerical point of view, several methods $[3,4,8,9,10,11,15$, $26,28]$ have been proposed.

We don't know if these methods are considered in real life applications outside laboratories, so we are proposing in this paper an unexpected real application of crack detection: the inpainting problem.

The goal of inpainting is to fill a hidden part of an image. In other words, if we denote by $\Omega$ the original image and $\omega$ the hidden part of the image, our goal is to recover the hidden part from the known part of the image in $\Omega \backslash \omega$. This problem has been widely studied. Mainly two methods have been considered:

- Extrapolation approach, the image is approximated in $\Omega \backslash \omega$, then the approximate function is evaluated in $\omega[31,32]$,

- Minimization of an energy cost function in $\omega$ based on a total variation norm [13, 14].

Crack detection allows to identify the edges of the hidden part of the image, the inpainting problem is then solved easily. Our crack detection technique [3] is based on the topological gradient approach [17, 20, 19, 25, 27, 29, 30].

Similar ideas have been applied by the authors to image restoration and classification $[6,21,5]$.

This papers is organized as follows. In section 2, we recall our method for crack localization. In section 3 , we present the adaptation of this technique to inpainting. Our crack localization technique gives satisfying results with incomplete data [21]; in section 4, we show that we are able to recover the image in $\omega$ when the intersection of its boundary with the boundary of $\Omega$ is not empty. We end the paper with some concluding remarks in section 5 .

\section{Crack localization problem}

\subsection{Dirichlet and Neumann problems}

We recall in this section the crack detection technique presented in [3]. Let $\Omega$ be a bounded open set of $\mathbb{R}^{2}$. We assume that there is a perfectly insulating crack $\sigma^{*}$ inside the domain. We consider the Poisson problem: We impose an input 
flux $\phi \in H^{-1 / 2}(\Gamma)$ on the boundary $\Gamma$ of $\Omega$, and we want to find $\sigma \subset \Omega$ such that the solution $u \in H^{1}(\Omega \backslash \sigma)$ to

$$
\begin{cases}\Delta u=0 & \text { in } \Omega \backslash \sigma \\ \partial_{n} u=\phi & \text { on } \Gamma \\ \partial_{n} u=0 & \text { on } \sigma\end{cases}
$$

satisfies $u_{\mid \Gamma}=T$ where $T$ is a given function of $H^{1 / 2}(\Gamma)$. In order to have a well-posed problem, we assume that

$$
\int_{\Gamma} \phi d s=0
$$

As we have an over-determination in the boundary conditions, we can define a Dirichlet and a Neumann problem:

$$
\text { Find } u_{D} \in H^{1}(\Omega \backslash \sigma) \text { such that } \begin{cases}\Delta u_{D}=0 & \text { in } \Omega \backslash \sigma, \\ u_{D}=T & \text { on } \Gamma, \\ \partial_{n} u_{D}=0 & \text { on } \sigma,\end{cases}
$$

and

$$
\text { find } u_{N} \in H^{1}(\Omega \backslash \sigma) \text { such that } \begin{cases}\Delta u_{N}=0 & \text { in } \Omega \backslash \sigma, \\ \partial_{n} u_{N}=\phi & \text { on } \Gamma, \\ \partial_{n} u_{N}=0 & \text { on } \sigma .\end{cases}
$$

It is clear that for the unknown crack $\sigma^{*}$, the two solutions $u_{D}$ and $u_{N}$ are equal. The idea is then to consider the following cost function:

$$
J(\sigma)=\frac{1}{2}\left\|u_{D}-u_{N}\right\|_{L^{2}(\Omega)}^{2},
$$

where $u_{D}$ and $u_{N}$ are solutions to problems (3) and (4) respectively for the given crack $\sigma$.

\subsection{Minimization by topological asymptotic analysis}

We consider in this section the two corresponding adjoint states, respectively solutions in $H^{1}(\Omega)$ to

$$
\left\{\begin{array}{l}
-\Delta v_{D}=-\left(u_{D}-u_{N}\right) \text { in } \Omega, \\
v_{D}=0 \text { on } \Gamma
\end{array}\right.
$$


and

$$
\left\{\begin{array}{l}
-\Delta v_{N}=+\left(u_{D}-u_{N}\right) \text { in } \Omega, \\
\partial_{n} v_{N}=0 \text { on } \Gamma .
\end{array}\right.
$$

The cost function $J(0)$ corresponds to the value of the cost function when no crack is inserted in the domain $\Omega$. In the following, we denote by $\bar{\sigma}$ a fixed bounded crack containing the origin $O$, and by $n$ a unit vector normal to this crak. We consider the insertion of a small crack at point $x: \sigma=x+\varepsilon \bar{\sigma}$, where $\varepsilon$ is supposed to be small.

The variation of the cost function induced by the insertion of this small crack is $J(\sigma)-J(0)$, and the topological gradient theory provides the following asymptotic expansion of $J$ when $\varepsilon$ tends to zero [3]:

$$
J(\sigma)-J(0)=f(\varepsilon) g(x, n)+o(f(\varepsilon)),
$$

where $f$ is a positive function depending only on the size of the crack, satisfying $\lim _{\varepsilon \rightarrow 0} f(\varepsilon)=0$, and where $g$ is called the topological gradient, depending only on the location of the inserted crack. The insertion of small cracks where $g$ is negative will hence minimize the cost function.

The topological gradient associated to the cost function $J$ defined in (5) is given by

$$
g(x, n)=-\left[\left(\nabla u_{D}(x) \cdot n\right)\left(\nabla v_{D}(x) \cdot n\right)+\left(\nabla u_{N}(x) \cdot n\right)\left(\nabla v_{N}(x) \cdot n\right)\right] .
$$

The topological gradient can then be rewritten in the following way

$$
g(x, n)=n^{T} M(x) n,
$$

where $M(x)$ is the $2 \times 2$ symmetric matrix defined by

$$
M(x)=-\operatorname{sym}\left(\nabla u_{D}(x) \otimes \nabla v_{D}(x)+\nabla u_{N}(x) \otimes \nabla v_{N}(x)\right) .
$$

We can deduce that $g(x, n)$ is minimal when the normal $n$ is the eigenvector associated to the smallest (i.e. most negative) eigenvalue of the matrix $M(x)$. In the following, this eigenvalue will be considered as the topological gradient.

We can then define a simple and very fast numerical algorithm. First, we solve the two direct problems (Dirichlet and Neumann), and the two corresponding 
adjoint problems. Then, at each point $x$ of the domain, we compute the matrix $M(x)$ and its two eigenvalues. The crack likely lies in the most negative gradient regions.

Applying the procedure described above, an example of location of the unknown cracks using the topological gradient is shown on figure 1 (extracted from [3]). The most negative values of the topological gradient are located around the actual cracks, and these results are obtained in only one iteration.
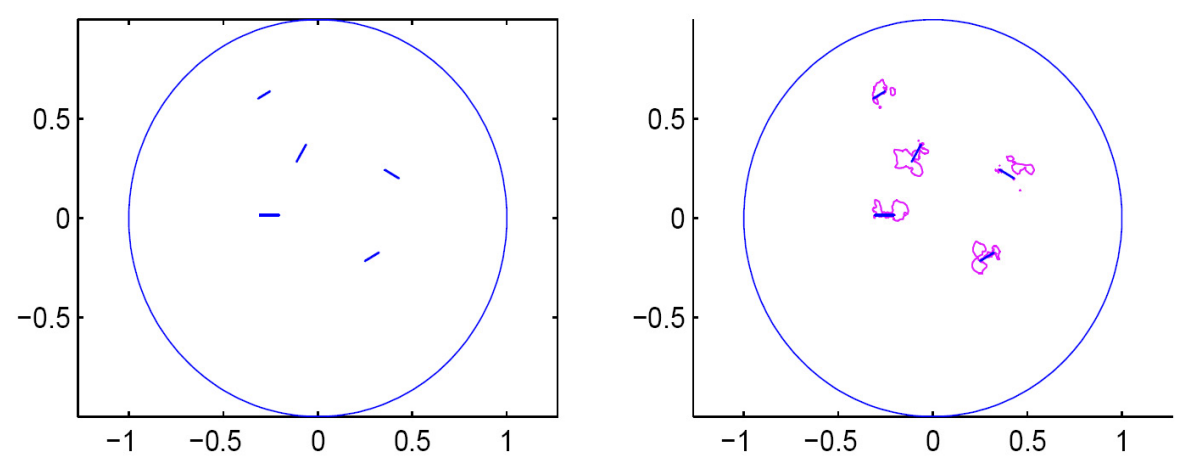

Figure 1 - Left: actual cracks; right: superposition of the actual cracks and a topological gradient isovalue. Figure extracted from [3].

\section{Application to inpainting problems}

\subsection{Algorithm}

We denote by $\Omega$ the image and $\Gamma$ its boundary, $\omega$ the missing part of the image and $\gamma$ its boundary. $v$ represents the image we want to restore, $T$ will represent here the value of the image on the boudary of the missing zone, and $\phi$ will be the corresponding flux. We have then $T=v$ and $\phi=\partial_{n} v$ in the corresponding domains. Theoretically, we have to assume $v$ to be enough regular, for example in $H^{2}(\Omega)$, but it will be possible to work with $v$ in $L^{2}(\Omega)$.

We now consider the problem of finding $u_{\alpha} \in H^{1}(\Omega)$, solution to the following equation:

$$
\left\{\begin{array}{l}
-\alpha \Delta u_{\alpha}+u_{\alpha} \cdot \chi_{\Omega \backslash \omega}=v \cdot \chi_{\Omega \backslash \omega} \text { in } \Omega, \\
\partial_{n} u_{\alpha}=0 \text { on } \Gamma
\end{array}\right.
$$


where $\alpha$ is a small positive number. This equation can be rewritten as

$$
\text { find } u_{\alpha} \in H^{1}(\Omega) \text { such that }\left\{\begin{array}{l}
-\alpha \Delta u_{\alpha}+u_{\alpha}=v \text { in } \Omega \backslash \omega, \\
\Delta u_{\alpha}=0 \text { in } \omega, \\
\partial_{n} u_{\alpha}=0 \text { on } \Gamma .
\end{array}\right.
$$

When $\alpha \rightarrow 0$, it is morally equivalent when $v$ is regular to find the solution $u_{N} \in H^{1}(\Omega \backslash \gamma)$ to

$$
\begin{cases}u_{N}=v & \text { in } \Omega \backslash \omega \\ \Delta u_{N}=0 & \text { in } \omega \\ \partial_{n} u_{N}=\partial_{n} v & \text { on } \gamma\end{cases}
$$

which can be seen as a Neumann problem.

From a numerical point of view, we will solve equation (13) with a very small positive $\alpha$ and we will consider that it is our Neumann problem. The Dirichlet problem does not require any special care.

The inpainting algorithm is then the following:

- Calculation of $u_{D}$ and $u_{N}$, respectively solutions to

$$
\begin{cases}u_{D}=v & \text { in } \Omega \backslash \omega \\ \Delta u_{D}=0 & \text { in } \omega \\ u_{D}=v & \text { on } \gamma\end{cases}
$$

where $u_{D} \in H^{1}(\Omega)$, and

$$
\left\{\begin{array}{l}
-\alpha \Delta u_{N}+u_{N}=v \quad \text { in } \Omega \backslash \omega \\
\Delta u_{N}=0 \quad \text { in } \omega \\
\partial_{n} u_{N}=0 \quad \text { on } \Gamma
\end{array}\right.
$$

where $u_{N}$ is in $H^{1}(\Omega)$ (the normal derivative is the same on the two sides of $\gamma$ ).

- Calculation of $v_{D}$ and $v_{N}$ the two corresponding adjoint states, respectively solutions to

$$
\left\{\begin{array}{l}
v_{D}=0 \text { in } \Omega \backslash \omega, \\
-\Delta v_{D}=-\left(u_{D}-u_{N}\right) \text { in } \omega, \\
v_{D}=0 \text { on } \gamma
\end{array}\right.
$$


and

$$
\left\{\begin{array}{l}
v_{N}=0 \quad \text { in } \Omega \backslash \omega, \\
-\Delta v_{N}=+\left(u_{D}-u_{N}\right) \quad \text { in } \omega, \\
\partial_{n} v_{N}=0 \text { on } \gamma .
\end{array}\right.
$$

- Computation of the $2 \times 2$ matrix $\mathrm{M}(\mathrm{x})$ (see eq. (11)) and its lowest eigenvalue $\lambda_{\min }$ at each point of the missing domain $\omega$.

- Definition of cracks localization: $\left\{x \in \omega ; \lambda_{\min }(x)<\delta<0\right\}$, where $\delta$ is a negative threshold.

- Calculation of $u$ solution to the Neumann problem (4) with this crack $\sigma$.

This algorithm has a complexity of $\mathcal{O}(n \cdot \log (n))$, where $n$ is the size of the image (i.e. number of pixels). See [5] for more details.

\subsection{Numerical results}

\subsubsection{Synthetic images}

We first consider a synthetic image, consisting of a black square on a white background. We assume that the center of the image is occluded. The missing region is represented by the grey square on image 2-a. A very fast approach for filling in the hole consists of solving the Laplace equation in the hole, with a Dirichlet boundary condition, i.e. the Dirichlet problem defined by equation (15). The corresponding solution is represented in figure 2-b.

We have then applied the algorithm defined in section 3.1, and figures 2-c and 2-d represent the identified cracks (or missing edges of the image) and the corresponding inpainted image respectively. We can notice that the corner of the black square is nearly identified.

Figure 3 represents the same images for the inpainting of a black circle on a white background. One can see that the missing part of the circle is very well identified. One may notice that even if the Dirichlet and Neumann conditions are almost the same in these two examples (square and circle), the reconstruction of the missing part is not the same. This is mainly due to the fact that the normal derivative of the Neumann solution is fully preserved at the interface $\gamma$ between 


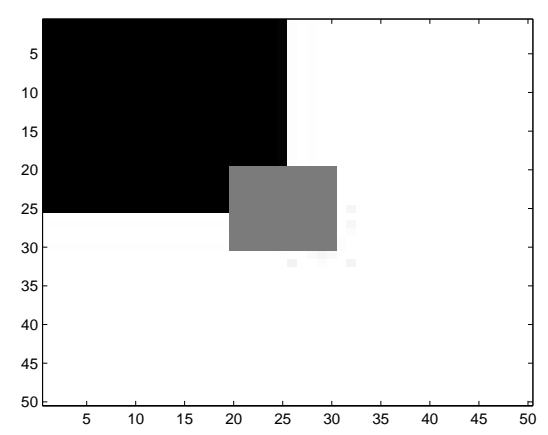

(a)

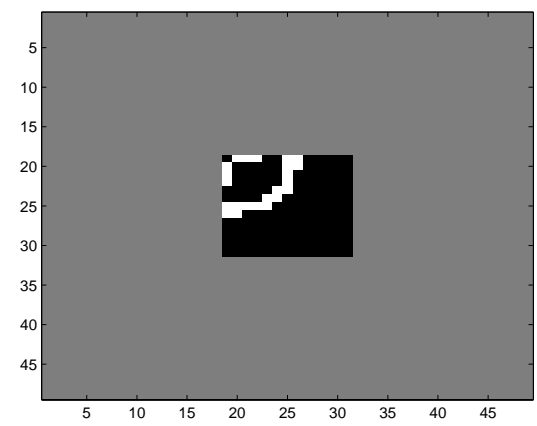

(c)

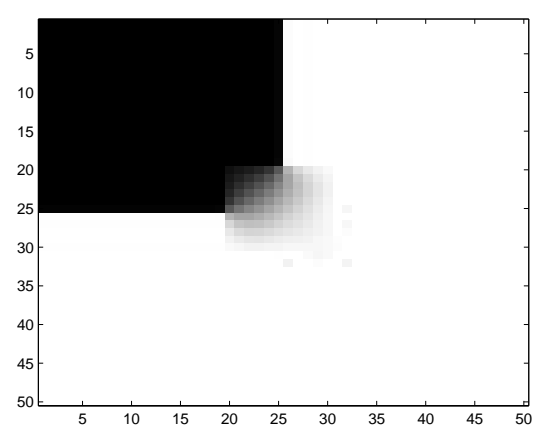

(b)

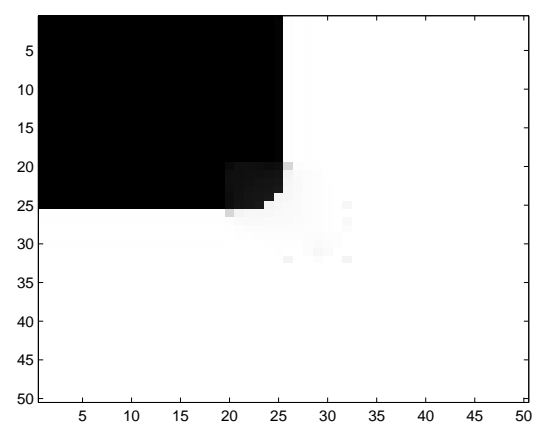

(d)

Figure 2 - Inpainting of a black square on a white background: occluded image (a), Laplace filled-in image (b), topological gradient (c) and inpainted image (d).

the hole $\omega$ and the image $\Omega \backslash \omega$, and hence the local geometry of the image is restored.

\subsubsection{Real images}

We have then applied our algorithm to the inpainting of a real image. Figure 4 shows respectively the image occluded by a black square (a), the filled-in image using the Laplace operator (b), the identified missing edges (c) and the corresponding inpainted image (d).

Figure 5 represents a zoom of figure 4 . One can see that the reconstruction of the missing part of the image is very satisfactory. Moreover, it is done in only one iteration of the topological gradient algorithm, which consists in 5 resolutions of a PDE (the two direct and two adjoint problems, and then one direct problem) 


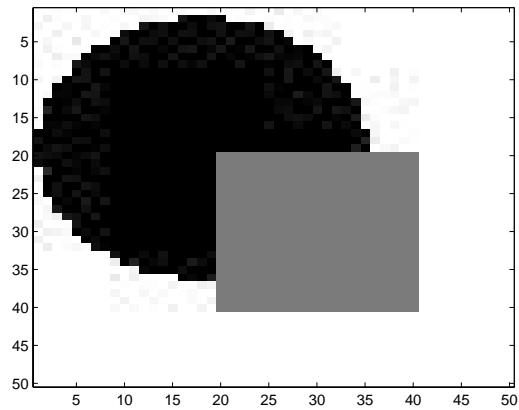

(a)

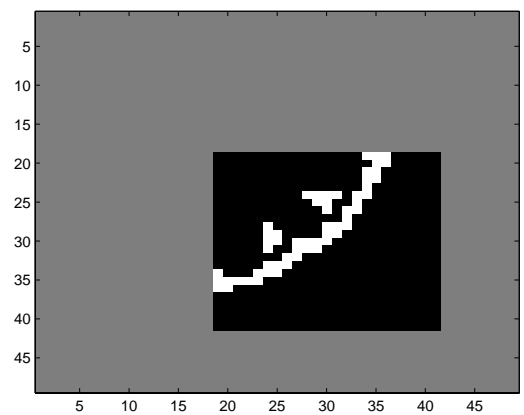

(c)

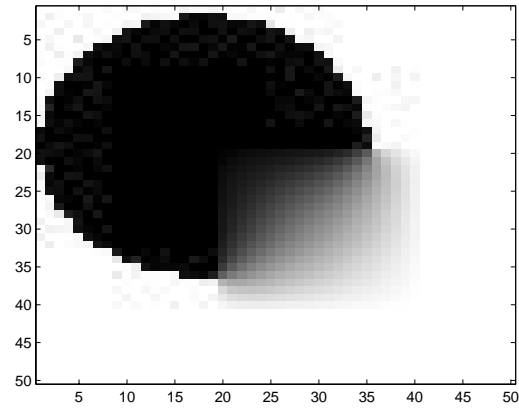

(b)

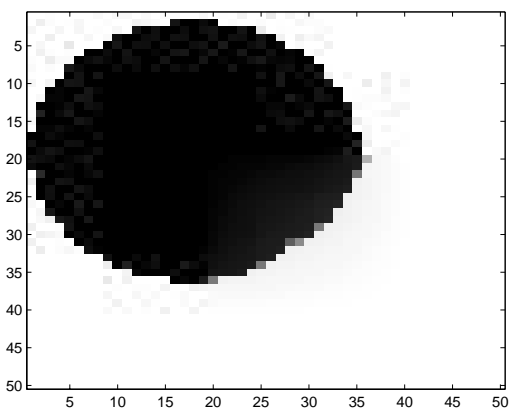

(d)

Figure 3 - Inpainting of a black circle on a white background: occluded image (a), Laplace filled-in image (b), topological gradient (c) and inpainted image (d).

on the domain $\Omega$ representing the image.

We now consider the occlusion of the same image by a black thick line. Figure 6 represents the results of our algorithm, and figure 7 represents a zoom of the same images. One can see that the image is still very well inpainted, even if the missing part is quite large. We may remark that we used the same threshold $\delta$ (see the algorithm in the previous section) for this whole zone, and it could be more interesting to look for valley lines of the topological gradient.

\section{Boundary inpainting}

In this section, we assume that the intersection of the inpainting domain and the boundary of the image is not empty, e.g. the inpainting domain is partly on the edge of the image. 


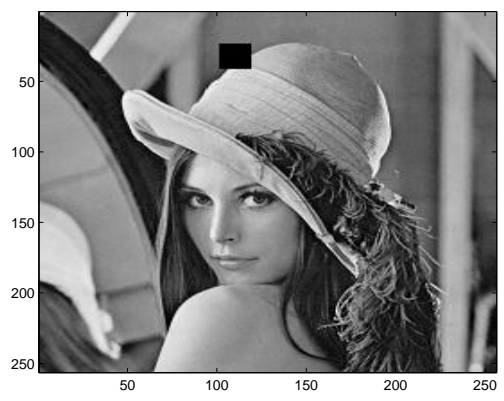

(a)

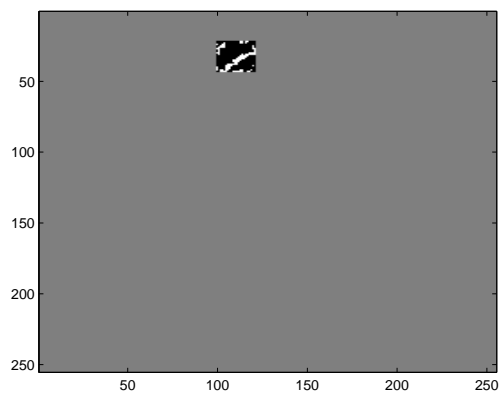

(c)

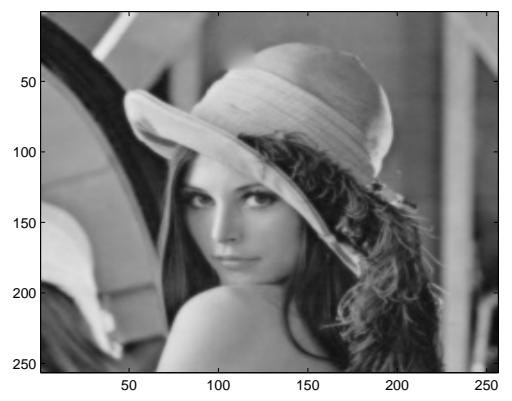

(b)

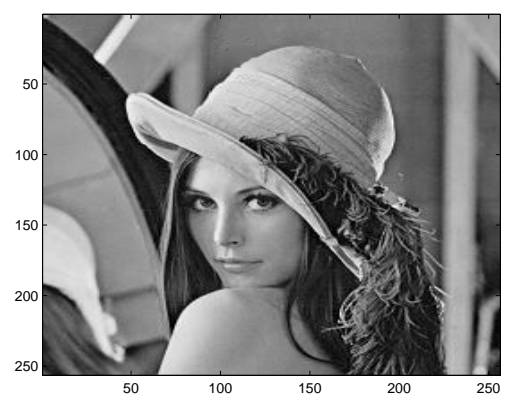

(d)

Figure 4 - Inpainting of a black square on a real image: occluded image (a), Laplace filled-in image (b), topological gradient (c) and inpainted image (d).

\subsection{Crack localization using incomplete data}

In comparison with section 2.1, we can assume that the input flux $\phi$ is still imposed on the whole boundary $\Gamma$ of $\Omega$, whereas the Dirichlet condition is incomplete: we assume that we measure $u$ on only a part $\Gamma_{0}$ of the boundary $\Gamma$. We denote by $\Gamma_{1}$ the complementary set in the boundary of $\Gamma_{0}: \Gamma=\Gamma_{0} \cup \Gamma_{1}$.

We can still solve the Neumann problem:

$$
\begin{cases}\Delta u_{N}=0 & \text { in } \Omega \backslash \sigma, \\ \partial_{n} u_{N}=\phi & \text { on } \Gamma, \\ \partial_{n} u_{N}=0 & \text { on } \sigma,\end{cases}
$$

but the Dirichlet problem defined by equation (3) can no more be solved, and it 


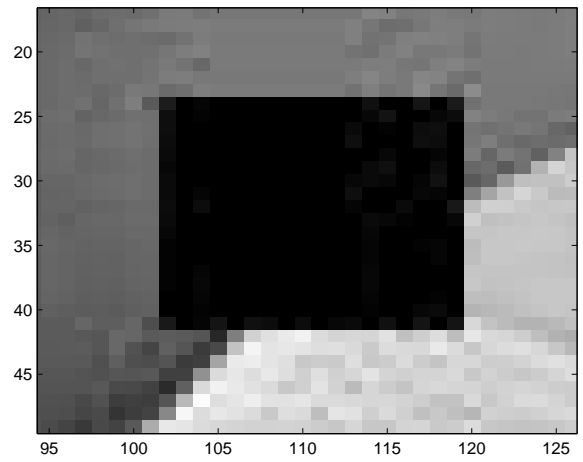

(a)

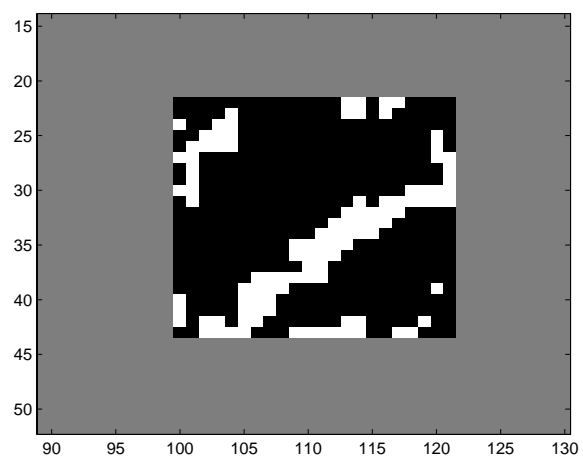

(c)

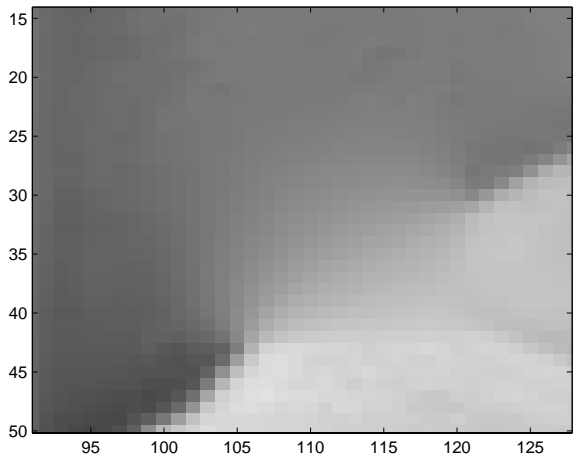

(b)

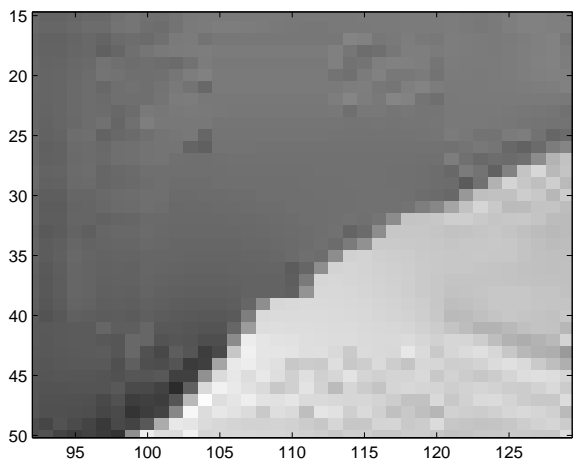

(d)

Figure 5 - Inpainting of a black square on a real image: zoom of occluded image (a), Laplace filled-in image (b), topological gradient (c) and inpainted image (d).

is replaced by the following Dirichlet-Neumann problem:

$$
\begin{cases}\Delta u_{D}=0 & \text { in } \Omega \backslash \sigma \\ u_{D}=T & \text { on } \Gamma_{0}, \\ \partial_{n} u_{D}=0 & \text { on } \Gamma_{1}, \\ \partial_{n} u_{D}=0 & \text { on } \sigma .\end{cases}
$$

We still consider the same cost function, measuring the discrepancy between these two solutions. The topological gradient remains unchanged, as well as the algorithm defined at the end of section 2.2.

Figure 8 (extracted from [3]) shows an example of localization of unknown cracks using the topological gradient, with incomplete data. In this example, $\Gamma_{0}$ 


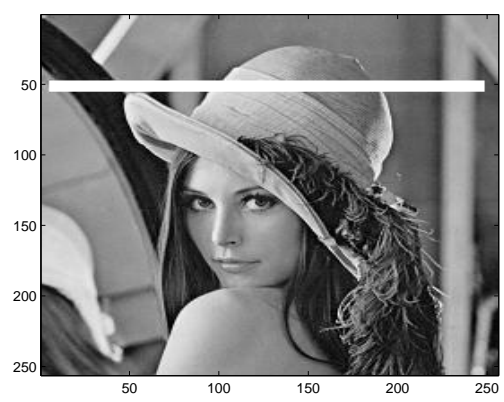

(a)

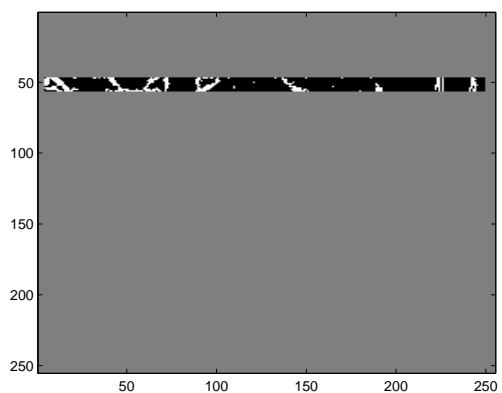

(c)

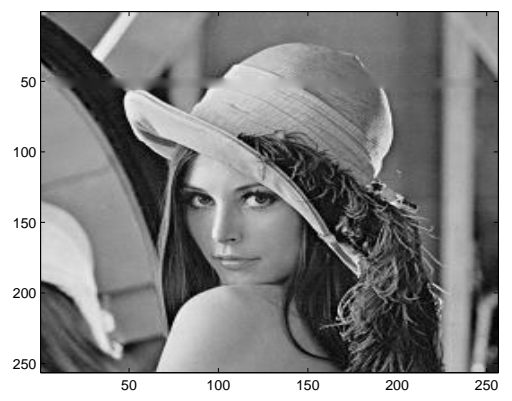

(b)

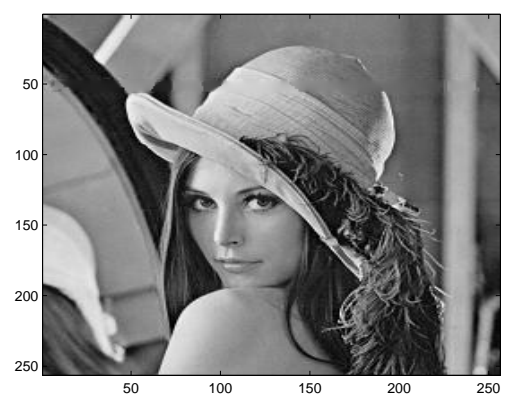

(d)

Figure 6 - Inpainting of a black line on a real image: occluded image (a), Laplace filled-in image (b), topological gradient (c) and inpainted image (d).

is the left half of the boundary, and $\Gamma_{1}$ is the right half of the boundary. The most negative values of the topological gradient are still located around the actual cracks.

\subsection{Boundary inpainting problem}

If we now consider the inpainting approach, using the same notations as previously defined, our problem is that $\gamma \cap \Gamma$ is not empty. We will denote by $\gamma_{1}$ this intersection, and $\gamma_{0}$ its complementary set in $\gamma$. Then, it is not possible to use a Dirichlet condition on all the boundary $\gamma$ for the Dirichlet problem, but only on $\gamma_{0}$. We will then consider the two following Dirichlet-Neumann and Neumann problems: 


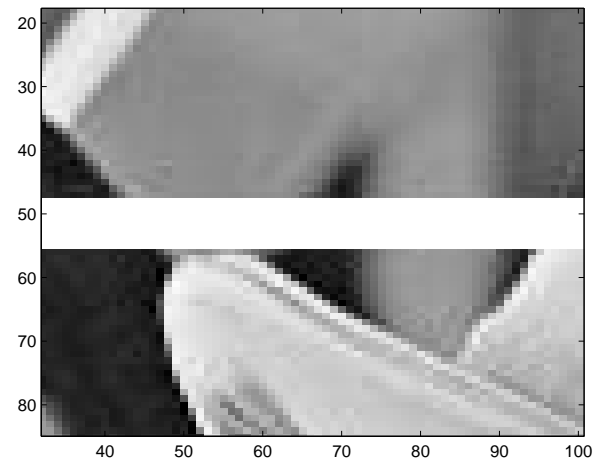

(a)

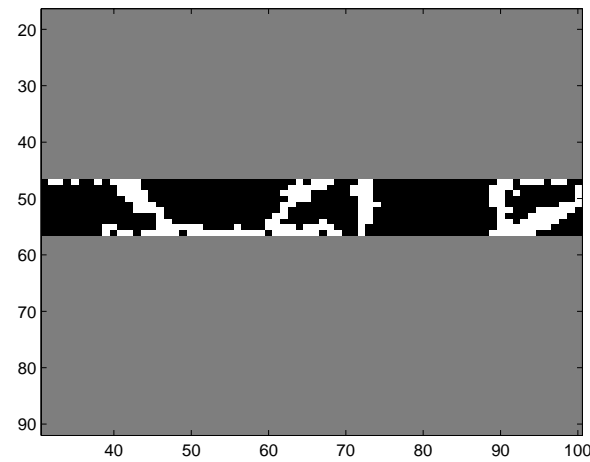

(c)

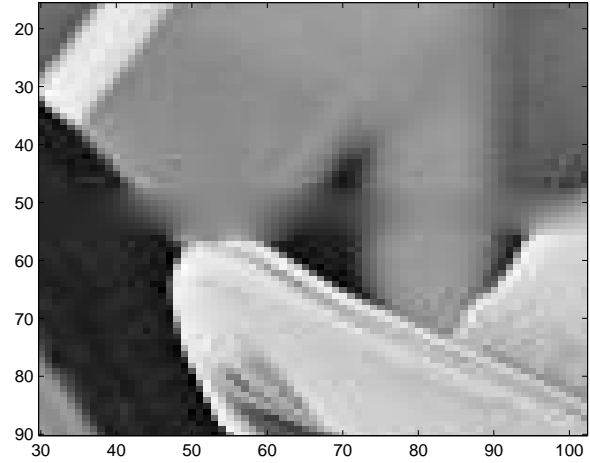

(b)

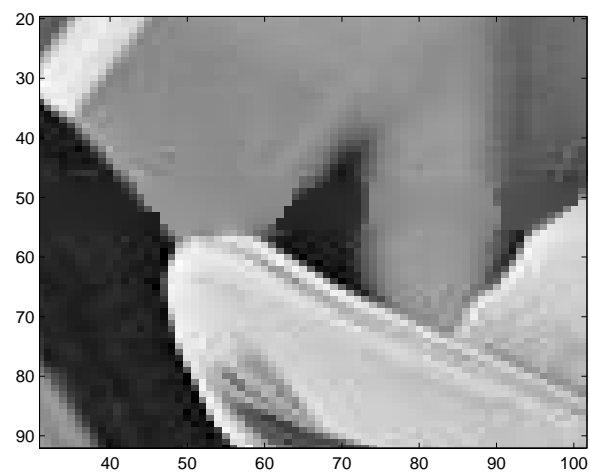

(d)

Figure 7 - Inpainting of a black line on a real image: zoom of occluded image (a), Laplace filled-in image (b), topological gradient (c) and inpainted image (d).

$$
\begin{cases}u_{D}=v & \text { in } \Omega \backslash \omega, \\ \Delta u_{D}=0 & \text { in } \omega, \\ u_{D}=v & \text { on } \gamma_{0}, \\ \partial_{n} u_{D}=0 & \text { on } \gamma_{1},\end{cases}
$$

and

$$
\left\{\begin{array}{l}
-\alpha \Delta u_{N}+u_{N}=v \quad \text { in } \Omega \backslash \omega, \\
\Delta u_{N}=0 \text { in } \omega, \\
\partial_{n} u_{N}=0 \text { on } \Gamma
\end{array}\right.
$$

where $\partial_{n} u_{N}$ is the same on the two sides of $\gamma_{0}$, and $\alpha$ is a small positive number. 

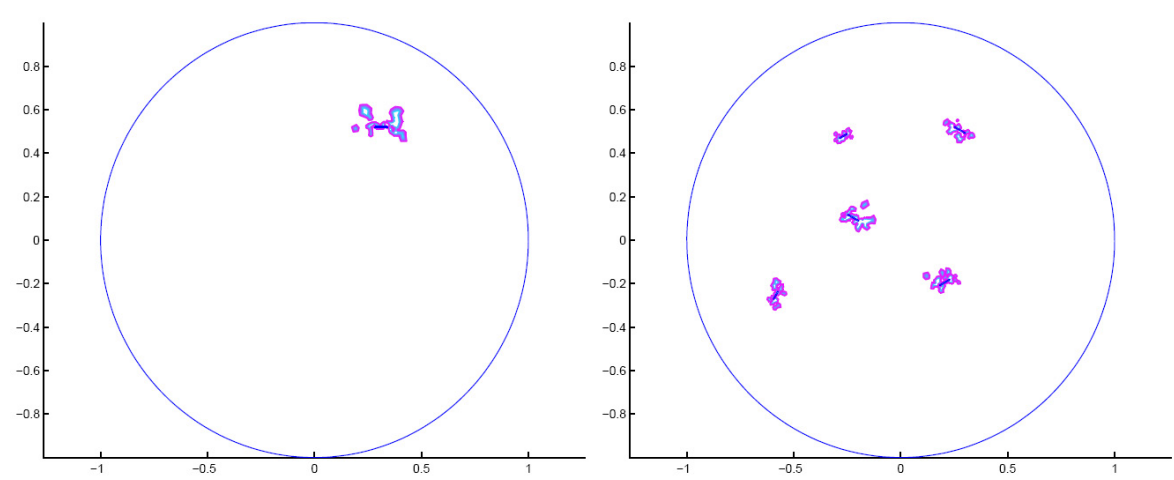

Figure 8 - Superposition of the actual cracks and a topological gradient isovalue with incomplete data. Figure extracted from [3].

The algorithm is then the same as in the previous section. We have then tried to fill in a real image occluded by a black square box located on the side of the image.

Figures 9-a,b,c,d represent respectively zooms of the occluded image, the Laplace filled-in image, the identified missing edges by the topological gradient, and the inpainted image. Figures 9-e and f represent the full size original and inpainted images. We can see that the results are almost the same as in the case of an occlusion inside the image: the missing edges are identified in only one iteration of the topological gradient, and the image is filled-in using only 5 resolutions of a PDE on the image.

\section{Conclusion}

The crack detection technique, based on topological gradient, provides an excellent frame for solving image processing problem. It has been applied to image restoration, image classification and image inpainting.

In all these cases, we obtained excellent results and the computing time is very short. Only $\mathcal{O}(n \log (n))$ operations are needed to solve the image processing problem, where $n$ is the size of the image (i.e.number of pixels). 


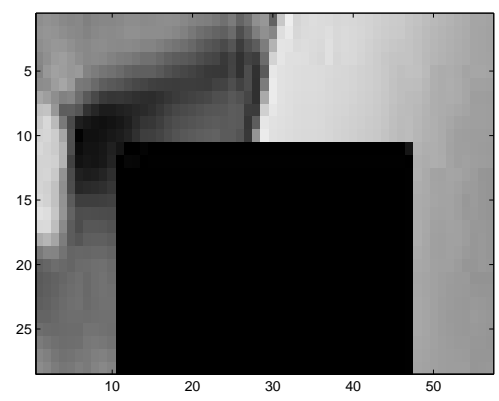

(a)

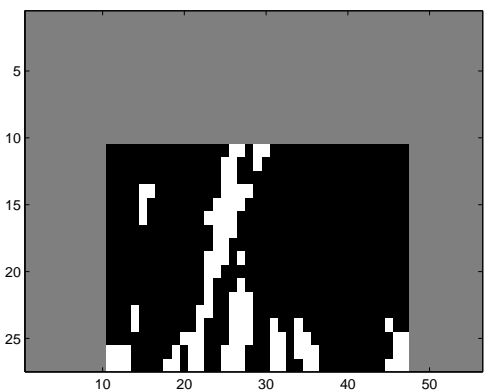

(c)

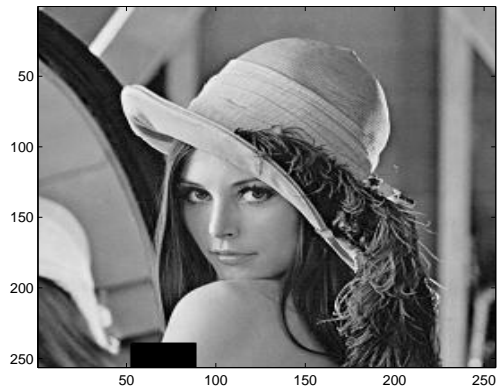

(e)

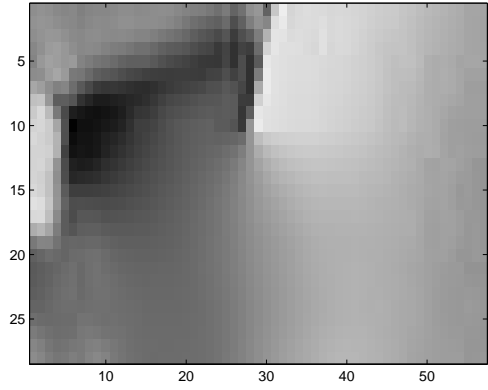

(b)

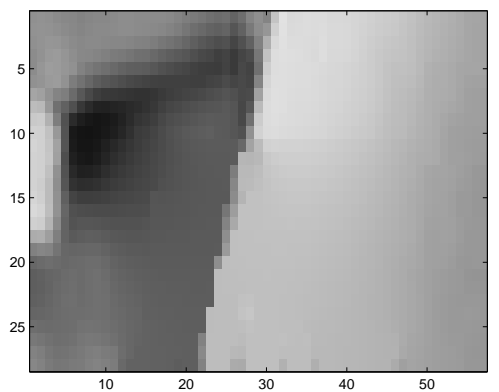

(d)

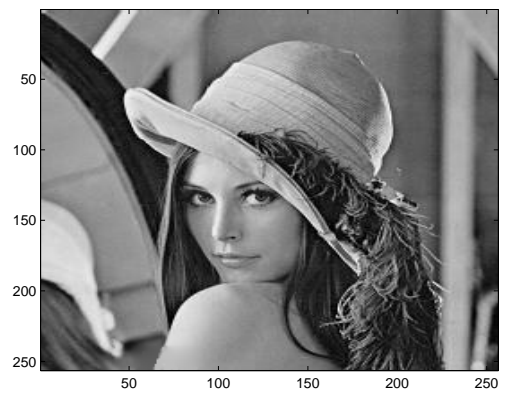

(f)

Figure 9 - Inpainting of a black square on the side of a real image: zoom occluded image (a), Laplace filled-in image (b), topological gradient (c) and inpainted image (d); full occluded image (e) and full inpainted image (f). 


\section{REFERENCES}

[1] G. Alessandrini, E. Beretta and S. Vessela, Determining linear cracks by boundary measurements: Lipschitz stability, SIAM J. Math. Anal., 27(2) (1996), pp. 361-375.

[2] G. Alessandrini and A. Diaz Valenzuela, Unique determination of multiple cracks by two measurements, SIAM J. Control Optim., 34(3) (1996), pp. 913-921.

[3] S. Amstutz, I. Horchani and M. Masmoudi, Crack detection by the topological gradient method, Control and Cybernetics, 34(1) (2005), pp. 119-138.

[4] S. Andrieux and A. Ben Abda, Identification of planar cracks by complete overdetermined data: inversion formulae, Inverse Problems, 12 (1996), pp. 553-563.

[5] D. Auroux, M. Masmoudi and L. Belaid, Image restoration and classification by topological asymptotic expansion, in Variational Formulations in Mechanics: Theory and Applications, E. Taroco, E.A. de Souza Neto and A.A. Novotny (Eds), CIMNE, Barcelona, Spain, in press (2006).

[6] L. Belaid, M. Jaoua, M. Masmoudi and L. Siala, Image restoration and edge detection by topological asymptotic expansion, C.R. Acad. Sci., Ser. I, 342(5) (2006), pp. 313-318.

[7] Z. Belhachmi and D. Bucur, Stability and uniqueness for the crack identification problem, SIAM J. Control Optim., to appear.

[8] A. Ben Abda, H. Ben Ameur and M. Jaoua, Identification of 2D cracks by elastic boundary measurements, Inverse Problems, 15 (1999), pp. 67-77.

[9] A. Ben Abda, M. Kallel, J. Leblond and J.-P. Marmorat, Line-segment cracks recovery from incomplete boundary data, Inverse Problems, 18 (2002), pp. 1057-1077.

[10] M. Bruhl, M. Hanke and M. Pidcock, Crack detection using electrostatic measurements, Math. Model. Numer. Anal., 35 (2001), pp. 595-605.

[11] K. Bryan and M.S. Vogelius, A review of selected works on crack identification, Proceedings of the IMA workshop on Geometric Methods in Inverse Problems and PDE Control, August 2001.

[12] A.P. Calderón, On an inverse boundary value problem, Seminar on Numerical Analysis and Its Applications to Continuum Physics (Rio de Janeiro, 1980), Soc. Brasil. Mat., Rio de Janeiro (1980), pp. 65-73.

[13] T. Chan and J. Shen, Mathematical Models for Local Deterministic Inpaintings, UCLA CAM Tech. Report 00-11, March (2000).

[14] T. Chan and J. Shen, Non-texture Inpainting by Curvature-driven Diffusions (CCD), UCLA CAM Tech. Report 00-35, Sept. (2000).

[15] A. Friedman and M.S. Vogelius, Determining cracks by boundary measurements, Indiana Univ. Math. J., 38(3) (1989), pp. 527-556.

[16] A. Friedman and M.S. Vogelius, Identification of small inhomogeneities of extreme con- 
ductivity by boundary measurements: a theorem of continuous dependence, Arch. Rational Mech. Anal., 105(4) (1989), pp. 299-326.

[17] S. Garreau, Ph. Guillaume and M. Masmoudi, The topological asymptotic for PDE systems: the elasticity case, SIAM J. Control. Optim., 39(6) (2001), pp. 1756-1778.

[18] J. Giroire and J.-C. Nédélec, Numerical solution of an exterior Neumann problem using a double layer potentiel, Mathematics of computation, 32(144) (1978), pp. 973-990.

[19] Ph. Guillaume and K. Sididris, Topological sensitivity and shape optimization for the Stokes equations, MIP Tech. Report 01-24, (2001).

[20] $\mathrm{Ph}$. Guillaume and K. Sididris, The topological asymptotic expansion for the Dirichlet problem, SIAM J. Control Optim., 41(4) (2002), pp. 1052-1072.

[21] L. Jaafar Belaid, M. Jaoua, M. Masmoudi and L. Siala, Application of the topological gradient to image restoration and edge detection, J. of Boundary Element Methods, to appear.

[22] A. Khludnev and V. Kovtunenko, Analysis of cracks in solids, WIT Press, SouthamptonBoston, 2000.

[23] R. Kohn and M. Vogelius, Relaxation of a variational method for impedance computed tomography, Comm. Pure Appl. Math., 40(6) (1987), pp. 745-777.

[24] S. Kubo and K. Ohji, Inverse problems and the electric potential computed tomography method as one of their application, in Mechanical Modeling of New Electromagnetic Materials, Elsevier Science Publisher, 1990.

[25] M. Masmoudi, The Topological Asymptotic, in Computational Methods for Control Applications, International Series GAKUTO, 2002.

[26] N. Nishimura and S. Kobayashi, A boundary integral equation method for an inverse problem related to crack detection, Int. J. Num. Methods Engrg., 32 (1991), pp. 1371-1387.

[27] B. Samet, S. Amstutz and M. Masmoudi, The topological asymptotic for the Helmholtz equation, SIAM J. Control. Optim., 42(5) (2003), pp. 1523-1544.

[28] F. Santosa and M. Vogelius, A computational algorithm to determine cracks from electrostatic boundary measurements, Int. J. Eng. Sci., 29 (1991), pp. 917-937.

[29] A. Schumacher, Topologieoptimisierung von Bauteilstrukturen unter Verwendung von Lopchpositionierungkrieterien, Thesis, Universitat-Gesamthochschule-Siegen, 1995.

[30] J. Sokolowski and A. Zochowski, On the topological derivative in shape optimization, SIAM J. Control Optim., 37 (1999), pp. 1241-1272.

[31] P. Wen, X. Wu and C. Wu, An Interactive Image Inpainting Method Based on RBF Networks, J. Wang et al. (Eds.), LNCS 3972, pp. 629-637, 2006.

[32] T. Zhou, F. Tang, J. Wang, Z. Wang and Q. Peng, Digital Image Inpainting with Radial Basis Functions, J. Image Graphics (In Chinese), 9(10) (2004), pp. 1190-1196. 